\title{
Using Cross-Cultural Studies to Improve Evidence on Dementia Prevention: Lessons from the Special Issue Sponsored by the International Research Network on Dementia Prevention (IRNDP)
}

\author{
M. Maria Glymour ${ }^{\mathrm{a}}$ and Rachel A. Whitmer ${ }^{\mathrm{b}}$ \\ ${ }^{a}$ Department of Epidemiology and Biostatistics, University of California, San Francisco, \\ San Francisco, CA, USA \\ ${ }^{\mathrm{b}}$ Department of Public Health Sciences, University of California, Davis, Davis, CA, USA
}

Accepted 1 July 2019

The manuscripts presented in this issue of the journal highlight the value of including diverse populations and settings in research on Alzheimer's disease and related disorders (ADRD). Evidence from populations typically underrepresented in ADRD research -including low- and middle-income countries (LMICs) as well as underrepresented groups in high-income countries-can offer greater scientific insight than evidence from populations already well studied. By integrating evidence from diverse settings, we can better address questions of causality and identify effective intervention strategies to reduce the burden of Alzheimer's disease.

This issue of the Journal of Alzheimer's Disease includes 8 articles drawing on cross-national or cross-cultural studies of Alzheimer's disease and related disorders (ADRD). Collectively, these

\footnotetext{
*Correspondence to: M. Maria Glymour, Department of Epidemiology and Biostatistics, University of California, San Francisco, San Francisco, CA 94158, USA. E-mail: maria.glymour@ ucsf.edu.
}

studies highlight several scientific advantages and challenges of broadening research on ADRD to include more diverse populations, settings, and study designs. Evidence from populations typically underrepresented in ADRD research-including low- and middle-income countries (LMICs) as well as underrepresented groups in high-income countries-holds promise for revealing successful intervention and prevention strategies. To capitalize on such opportunities, however, we need thoughtful design and data harmonization and analysis approaches.

The era of evaluating ADRD "risk factors" without rigorous consideration of causality is over. Causal understanding provides the path to effective prevention and treatment. In many cases we have several studies showing that a risk factor is associated with or predictive of ADRD, yet substantial debate about causality remains. This fundamental challenge is implicit in recent high profile estimates of the fraction of ADRD that could be prevented, because all such estimates rely on the assumption that we have accurate assessments of causal effects [1]. To identify the 
causal determinants of ADRD, we will need novel research approaches. Although individual studies are unlikely to provide conclusive evidence about causal effects, we can carefully knit together evidence from different study designs and research settings to evaluate alternative causal structures. This process has been referred to as "triangulation" and offers the best promise for learning about causal structures from observational data [2-4]. Triangulation recognizes that no study is perfect, but aims to identify alternative study designs with different, unrelated weaknesses. By combining results from different approaches, the overall strength of evidence gains strength. For example, a very strong association between education and cognitive test scores has been documented in numerous high-income countries. The major limitations in these studies is the difficulty of ruling out the confounding influence of individual characteristics such as intelligence or childhood socioeconomic status that may influence both schooling and late-life cognition. Evidence from studies in settings with very different confounders [5], or based on natural experiments that could not plausibly be influenced by individual characteristics [6], strengthens the overall evidence base, even if the new studies have different limitations.

Cross-national studies, especially research drawing on LMICs, are particularly valuable in efforts to evaluate causality. Cross-national comparisons offer a potential solution to the problem of disentangling confounders from causal factors: confounding bias that is intractable in one country may be completely different in other settings, offering opportunities to triangulate on the true effects. This phenomenon means that evidence from new populations often has greater potential scientific impact than evidence from well-studied communities. Although in some cases homogeneity has been argued to be an advantage for individual studies, when considering ADRD research as a whole, diversity and heterogeneity of populations and designs will be more powerful and enlightening. Additional costs are entailed in fielding studies in low-resource settings or recruiting individuals from underrepresented communities into ADRD studies, so recognizing that such participants also provide additional benefit is important. Ignoring LMICs is a missed opportunity for revealing the underlying etiology of ADRD and determinants of outcomes experienced by people living with ADRD.

If we are to take best advantage of newly emerging evidence from LMICs, cross-national studies, or numerous small studies in diverse populations, we must harmonize our research studies and adopt more formal approaches to integrating evidence. Harmonization is the process of making data from different studies comparable so the evidence from multiple sources can be combined. This means harmonizing outcome assessments, exposure assessments, and analytic approaches in future research and finding ways to cross-walk between results from existing studies that were not harmonized in advance. Both non-statistical methods such as identifying shared items and statistical methods such as latent variable modeling are relevant for harmonization [7, 8]. Differences in cultural context create additional challenges in harmonizing surveys, but these challenges are unavoidable: we must grapple with the role of cultural context eventually if we are to field interventions in diverse settings. The Health and Retirement Study international sister studies have been extremely successful at identifying shared data elements that could appropriately be fielded in numerous international settings [9]. The international replications of the Finnish Geriatric Intervention Study to Prevent Cognitive Impairment and Disability (FINGERS) trial are also inevitably grappling with how to operationalize this multi-component intervention in diverse settings and achieve harmonized outcome assessments to facilitate integration of results [10].

Without this harmonization, we will learn much less from each individual study and we will never be able to draw generalized conclusions about the drivers of disease. Careful harmonization is essential to allow meaningful comparison across studies and therefore to identify factors that attenuate or exacerbate effects of a risk factor. Without rigorous harmonization, when we find different effect estimates in different studies, we will not know if these differences are substantively important or merely artefacts of measurement or design differences.

The relevance of the lifecourse is another theme from these papers, and recognition that lifecourse milestones and stages may differ across settings. To identify causal determinants of ADRD, our research must incorporate more explicit models of the years- or perhaps decades-long development of AD. We have long understood that dementia typically emerges after years of accumulating disease, and it therefore stands to reason that the effects of exposure will depend on when an individual is exposed. This is a fundamental challenge when evaluating time-varying exposures, such as body mass index (BMI), social connections, behavioral patterns, or even modifiable "omic" measures, such as proteomics, but developmentally 
specific effects of exposures must be evaluated if we are to design successful interventions.

These points are well illustrated in the interesting array of studies in this issue of the journal. Danat et al. [11] present a meta-analysis of studies evaluating the association between BMI and BMI categories with dementia risk, focusing on community-based studies that assessed BMI among people ages 65+. In this fascinating report, they find that among studies with less than 9 years of follow-up, the overall metaanalyzed relative risk per BMI point is $0.95(95 \%$ CI: $0.93,0.96)$, with a non-significant $\mathrm{I}^{2}$ statistic of $21 \%(p=0.25)$, indicating most of the heterogeneity between studies could be attributed to sampling variability. In contrast, in studies with 9 or more years of follow-up, they found a meta-analyzed RR of $1.03(0.96,1.11)$, with an $\mathrm{I}^{2}$ of $75 \%(p<0.001)$, indicating that most of the variability was likely due to real differences in effects from different studies. This intriguing result illustrates the importance of studies across diverse contexts: there are major modifiers of the effects of even extremely well-studied risk factors such as BMI. These modifiers may relate to age of the sample, duration of the follow-up or contextual factors such that BMI represents distinct physiologic or social risk. Danat et al. [11] highlight the potential for reverse causation: it is very likely that changes in BMI in late life are primarily consequences of disease, rather than determinants, and therefore not useful targets for preventive intervention. A key role for meta-research such as the Danat paper is to identify research gaps. All 16 previously published studies identified in their review were from high-income countries, and they added valuable new results from China. These data from Chinese adults showed a qualitative difference in the association between BMI and dementia risk for men (among whom overweight/obesity predicted higher risk of dementia) compared to women (among whom overweight/obesity predicted lower risk of dementia). New studies in LMICs or other settings with different social patterning of BMI or distinct timing of the obesity epidemic may offer additional leverage for causal identification.

Peters et al. [12] identified 13 cohort studies on air pollution and ADRD, all from high income settings. They considered studies of either incident dementia or rate of cognitive change and found consistent evidence that exposure to particulate matter $\leq 2.5 \mathrm{~m}$, nitrogen dioxide, nitrous oxides, and carbon monoxide predicted increased risk of dementia or accelerated cognitive decline. Their review illustrates the challenge of integrating findings from studies with different but clearly related outcome measures, such as cognitive decline and dementia incidence. Environmental determinants of dementia risk, including air pollution, may have tremendous public health consequences because, as Peters et al. note, air pollution is "pervasive, global, life-long" and modifiable. Regulation of air pollutants is already common, and rigorous evidence on effects on dementia risk can help guide the development of those regulations. Peters et al. [12] lay out priority areas for future research, including improved exposure measures, evaluating exposure duration, and understanding how effects differ across settings. Individuals in LMICs face a major burden of premature mortality from air pollution [13], and yet, LMICs were notably missing from the extant literature. Environmental health issues are likely to be extremely relevant to ADRD risk in LMICs. For example, as Peters notes, prior autopsy studies in Mexico City found that exposure to urban air pollution predicted a greater neuropathologic burden even among children and young adults [14]. Developing a foundation of evidence on environmental determinants of ADRD risk is a high priority topic for global dementia research.

Evans et al. [15] identified sixty-five articles on aspects of social isolation and cognitive function or dementia risk in older adults, restricting to studies with at least one year of follow-up after the measurement of social isolation. Social relationships and the downstream consequences are complex to measure. Comprehensive assessments are typically based on long questionnaires and rarely fielded in large population based studies (with some notable exceptions [16]), thus most research on social ties and cognitive function draws on brief summary reports of engagement in social activities, number of close ties, or self-assessed adequacy of social support. In the Evans review [15], they found fairly consistent associations of social activity or networks with cognitive outcomes, regardless of the duration of follow-up time, gender of respondent, or outcome domain. As the authors note, there are several caveats, in that incipient ADRD almost certainly leads to social withdrawal years prior to diagnosis. They also found evidence of publication bias, indicating that results consistent with expectation are more likely to be published. Given the large number of studies to date using similar research designs to evaluate the links between social activity and ADRD risk, the most valuable future directions in this area will adopt novel designs to circumvent major challenges 
to establishing causality. This might include quasiexperimental approaches or, as Evans et al. [15] notes, randomized interventions. Because there have been numerous randomized studies intervening on social experiences for individuals in diverse settings, such as caregivers or patients with various diagnoses [17, 18], such efforts might leverage existing studies by incorporating new cognitive measures.

Radford et al.'s study [19] of factors associated with dementia in a sample of 336 Aboriginal Australians aged $60+$ years illustrates both the promise and challenges of focusing research attention in communities rarely represented in dementia studies. Previous work indicates Aboriginal Australians have substantially higher dementia risk than other Australians [20], and although the existing evidence base is limited, this demonstrates the relevance of ADRD for Aboriginal health. Radford et al. [19] identified social (childhood trauma and low-skill occupation), behavioral (past high-risk alcohol use) and medical conditions (stroke, head injury) as significant predictors of prevalent dementia. These results are not surprising and indicate general consistency with dementia predictors in other studies, although Radford et al. [19] note that education in this sample was not independently associated with dementia. More specifically, the association between education and dementia was not statistically significant, although the effect estimate $(\mathrm{OR}=0.82)$ reported by Radford was protective similar to estimates reported elsewhere, but not precisely estimated. In other words, the association in their data was not statistically significant, but an effect size indicating an $18 \%$ reduction in odds of dementia would be substantively important if it were precisely estimated. This is an intrinsic challenge in studying hard-to-reach populations: the studies are often expensive and typically face major resource constraints. This calls not only for more resources for this research, but also for a consideration of best statistical methods for evaluating familiar risk factors in novel populations. In such studies, we must carefully consider our null hypothesis: instead of testing the null that a risk factor has no effect for each population, should we begin with the assumption that effects are the same in all populations and test whether effects differ? If we pursue the standard approach of testing all effects against the null hypothesis of no effect, we risk systematically missing important risk factors in hard-to-reach populations. Another key point brought to light by Radford et al is that it may be impossible to identify the major determinants of dementia in a population by fielding a study within only that population. As Rose's classic work beautifully illustrated, if everyone within a population is exposed to a particular risk factor, it is impossible to show the role of that risk factor unless it can be compared to a population with less exposure [21]. This is particularly important in extremely disadvantaged communities, in which poor quality education, exposure to discrimination, experiences of trauma or other social risks may be nearly universal. By combining studies across diverse settings, we have the potential to learn much more.

Using data from 160 Malay individuals, grouped into four age brackets $(30-39,40-49,50-59$, and 0-69), Abu Bakar et al. [22] apply proteomic approaches to evaluate which protein profiles may differ by age in hopes to gain insight into proteomic patterns linked to cognitive decline. One notable aspect of this paper is the use of pooled samples for proteomic analyses. This approach merits special note because it makes a potentially very expensive analysis more feasible while, if correctly implemented, sacrificing little in terms of evidence quality [23]. Importantly, pooling samples heterogeneous on a risk factor makes it nearly impossible to evaluate the effects of that particular risk factor (e.g., pooling samples for men and women precludes evaluation of the effects of sex on the biomarker of interest in the sample), so detailed advance planning about the analyses of interest is important.

In a large $(n=4,128)$ sample of Australians, Sacre et al. [24] found that the $3.4 \%$ of the population with albuminuria had accelerated declines in both memory and processing speed. Less than $1 \%$ of the sample $(n=39)$ had frank kidney dysfunction (eGFR $<60 \mathrm{ml} / \mathrm{min} / 1.73 \mathrm{~m}^{2}$ ), so it is not surprising that they detected no significant association between kidney dysfunction and cognitive decline. Their paper takes advantage of the repeated measures of kidney function to assess the duration of albuminuria required to see an effect on cognition. They find that although albuminuria measured 12 years prior to memory was significantly associated, albuminuria measured only 7 years earlier had almost no association with memory. These results should be replicated in an independent sample but highlight that many risk factors likely to causally impact disease may take over a decade to do so, requiring substantial follow-up to make headway on causal determinants.

Cherbuin et al. [25] used data from the influential 10/66 study to evaluate the relationship between chronic obstructive pulmonary disease (COPD) and risk of incident dementia over 3-years of follow-up. 
They found that although COPD was a strong predictor of mortality, it was significantly associated with dementia only in Cuba (out of 7 countries evaluated). Cherbuin et al. [25] undertook formal efforts to address the clear potential bias introduced because COPD increases mortality. Survival bias is a pervasive problem in dementia research, and it is receiving increasing formal attention [26, 27]. The Fine and Gray method adopted by Cherbuin et al. [25] is useful in particular for public health planning about potential future burden of disease [28], though less informative about disease etiology.

Deckers et al. [29] used data from the Dutch Doetinchem Cohort Study to evaluate the relationship between the 'LIfestyle for BRAin Health' (LIBRA) score and cognitive change and indirectly address the controversial topic of gender differences in dementia risk. The LIBRA score, which combines five behavioral measures (diet, alcohol use, cognitive activity, physical activity, and smoking) with seven disease indicators (coronary heart disease, diabetes, hypercholesterolemia, hypertension, depression, obesity, and renal disease). Although describing renal disease, depression, and other complex, life-threatening diseases as "lifestyle" factors is a misnomer, the LIBRA index provides a powerful summary of several risk factors that might be prevented by structural, behavioral, or medical intervention. Deckers et al. [29] found that worse LIBRA scores predicted slightly faster decline in cognitive flexibility and mental speed, with no apparent differences by education level. LIBRA predicted verbal memory decline among women but not men, although given that small differences were observed in only one cognitive outcome, Deckers et al. [29] summarized the evidence as roughly similar associations by education and gender. The possibility of gender differences in ADRD incidence has received substantial research attention, but recent work also highlights the many reasons that small differences may reflect methodological artefacts [30]. The careful sensitivity analyses evaluating the role of cardiovascular disease and baseline differences in cognitive test scores help allay concerns that their these factors may have contributed to their findings [29].

In combination, these studies illustrate the promise of international or cross-national ADRD research and efforts to include often underrepresented populations in ADRD research. Alongside the moral imperative of increasing diversity in ADRD research, we should add the scientific imperative. Understanding the biology of ADRDs and the causal pathways which could be targeted to prevent the devastating cognitive consequences has proven devilishly difficult. Evidence from novel research settings can broaden our vision of prevention strategies and circumvent persistent research challenges.

\section{ACKNOWLEDGMENTS}

The authors gratefully acknowledge financial support from the US National Institute on Aging (RF1AG052132).

Authors' disclosures available online (https:// www.j-alz.com/manuscript-disclosures/19-0304r1).

\section{REFERENCES}

[1] Livingston G, Sommerlad A, Orgeta V, Costafreda SG, Huntley J, Ames D, Ballard C, Banerjee S, Burns A, CohenMansfield J (2017) Dementia prevention, intervention, and care. Lancet 390, 2673-2734.

[2] Lawlor DA, Tilling K, Davey SG (2017) Triangulation in aetiological epidemiology. Int J Epidemiol 45, 1866.

[3] Vandenbroucke JP, Broadbent A, Pearce N (2016) Causality and causal inference in epidemiology: The need for a pluralistic approach. Int J Epidemiol 45, 1776-1786.

[4] Lipsitch M, Tchetgen ET, Cohen T (2010) Negative controls: A tool for detecting confounding and bias in observational studies. Epidemiology 21, 383-388.

[5] Kobayashi LC, Glymour MM, Kahn K, Payne CF, Wagner RG, Montana L, Mateen FJ, Tollman SM, Berkman LF (2017) Childhood deprivation and later-life cognitive function in a population-based study of older rural South Africans. Soc Sci Med 190, 20-28.

[6] Hamad R, Elser H, Tran DC, Rehkopf DH, Goodman SN (2018) How and why studies disagree about the effects of education on health: A systematic review and meta-analysis of studies of compulsory schooling laws. Soc Sci Med 212, 168-178.

[7] Gross AL, Sherva R, Mukherjee S, Newhouse S, Kauwe JS, Munsie LM, Waterston LB, Bennett DA, Jones RN, Green RC (2014) Calibrating longitudinal cognition in Alzheimer's disease across diverse test batteries and datasets. Neuroepidemiology 43, 194-205.

[8] Abner EL, Schmitt F, Nelson P, Lou W, Wan L, Gauriglia R, Dodge H, Woltjer R, Yu L, Bennett D (2015) The Statistical Modeling of Aging and Risk of Transition Project: Data collection and harmonization across 11 longitudinal cohort studies of aging, cognition, and dementia. Obs Stud 1, 56.

[9] Suzman R, Beard JR, Boerma T, Chatterji S (2015) Health in an ageing world-what do we know? Lancet 385, 484-486.

[10] Kivipelto M, Mangialasche F, Ngandu T, Eg JJE, Martín N, Soininen H, Tuomilehto J, Lindström J, Solomon A, Mangialasche F (2018) World Wide Fingers will advance dementia prevention. Lancet Neurol 17, 27.

[11] Danat IM, Clifford A, Partridge M, Zhou W, Bakre AT, Chen A, McFeeters D, Smith T, Wan Y, Copeland J, Anstey KJ, Chen R (2019) Impacts of overweight and obesity in older age on the risk of dementia: A systematic literature review and a meta-analysis. J Alzheimers Dis 70, S87-S99. 
[12] Peters R, Ee N, Peters J, Booth A, Mudway I, Anstey KJ (2019) Air pollution and dementia: A systematic review. $J$ Alzheimers Dis 70, S145-S163.

[13] Cohen AJ, Brauer M, Burnett R, Anderson HR, Frostad J, Estep K, Balakrishnan K, Brunekreef B, Dandona L, Dandona R (2017) Estimates and 25-year trends of the global burden of disease attributable to ambient air pollution: An analysis of data from the Global Burden of Diseases Study 2015. Lancet 389, 1907-1918.

[14] Calderón-Garcidueñas L, Gónzalez-Maciel A, ReynosoRobles R, Delgado-Chávez R, Mukherjee PS, Kulesza RJ, Torres-Jardón R, Ávila-Ramírez J, Villarreal-Ríos R (2018) Hallmarks of Alzheimer disease are evolving relentlessly in Metropolitan Mexico City infants, children and young adults. APOE4 carriers have higher suicide risk and higher odds of reaching NFT stage V at $\leq 40$ years of age. Environ Res 164, 475-487.

[15] Evans IEM, Martyr A, Collins R, Brayne C, Clare L (2019) Social isolation and cognitive function in later life: A systematic review and meta-analysis. J Alzheimers Dis $\mathbf{7 0 ,}$ S119-S144.

[16] Bearman PS, Moody J, Stovel K (2016) Chains of affection: The structure of adolescent romantic and sexual networks. In Handbook of Applied System Science, Neal ZP, ed. Taylor \& Francis, pp. 164-190.

[17] Dam AE, de Vugt ME, Klinkenberg IP, Verhey FR, van Boxtel MP (2016) A systematic review of social support interventions for caregivers of people with dementia: Are they doing what they promise? Maturitas 85, 117-130.

[18] Coll-Planas L, Nyqvist F, Puig T, Urrútia G, Solà I, Monteserín R (2017) Social capital interventions targeting older people and their impact on health: A systematic review. $J$ Epidemiol Community Health 71, 663-672.

[19] Radford K, Lavrencic LM, Delbaere K, Draper B, Cumming R, Daylight G, Mack HA, Chalkley S, Bennett H, Garvey G, Hill TY, Lasschuit D, Broe GA (2019) Factors associated with the high prevalence of dementia in older Aboriginal Australians. J Alzheimers Dis 70, S75-S85.

[20] Smith K, Flicker L, Lautenschlager N, Almeida O, Atkinson D, Dwyer A, LoGiudice D (2008) High prevalence of dementia and cognitive impairment in Indigenous Australians. Neurology 71, 1470-1473.

[21] Rose G (2001) Sick individuals and sick populations. Int J Epidemiol 30, 427-432.
[22] Abu Bakar ZH, Damanhuri HA, Makpol S, Wan Kamaruddin WMA, Abdul Sani NF, Amir Hamzah AIZ, Nor Aripin KN, Rani MDM, Azila Noh N, Razali R, Mazlan M, Abdul Hamid H, Mohamad M, Wan Ngah WZ (2019) Effect of age on the protein profile of healthy Malay adults and its association with cognitive function competency. J Alzheimers Dis 70, S43-S62.

[23] Schisterman EF, Vexler A (2008) To pool or not to pool, from whether to when: Applications of pooling to biospecimens subject to a limit of detection. Paediatr Perinat Epidemiol 22, 486-496.

[24] Sacre JW, Magliano DJ, Zimmet PZ, Polkinghorne KR, Chadban SJ, Anstey KJ, Shaw JE (2019) Associations of chronic kidney disease markers with cognitive function: A 12-year follow-up study. J Alzheimers Dis 70, S19-S30.

[25] Cherbuin N, Walsh EI, Prina AM (2019) Chronic obstructive pulmonary disease and risk of dementia and mortality in lower to middle income countries. J Alzheimers Dis $\mathbf{7 0}$, S63-S73.

[26] Mayeda E, Tchetgen Tchetgen EJ, Power M, Weuve J, Jacqmin-Gadda H, Marden J, Vittinghoff E, Keiding N, Glymour MM (2016) A simulation platform to quantify survival bias: An application to research on determinants of cognitive decline. Am J Epidemiol 184, 378-387.

[27] Weuve J, Tchetgen Tchetgen E, Glymour MM, Beck TL, Aggarwal NT, Wilson RS, Evans DA, Mendes de Leon CF (2012) Accounting for bias due to selective attrition in analyses of cognitive decline: The example of smoking and cognitive decline in older adults. Epidemiology 23, 119-128.

[28] Fine JP, Gray RJ (1999) A proportional hazards model for the subdistribution of a competing risk. J Am Stat Assoc 94, 496-509.

[29] Deckers K, Nooyens A, van Boxtel M, Verhey F, Verschuren M, Köhler S (2019) Gender and educational differences in the association between lifestyle and cognitive decline over 10 years: The Doetinchem Cohort Study. J Alzheimers Dis 70, S31-S41.

[30] Mayeda ER (2019) Examining sex/gender differences in risk of Alzheimer's disease and related dementias: Challenges and future directions. Am J Epidemiol 188, 1224-1227. 